\title{
A Retinal Ganglion Cell That Can Signal Irradiance Continuously for 10 Hours
}

\author{
Kwoon Y. Wong \\ Department of Ophthalmology \& Visual Sciences and Department of Molecular, Cellular \& Developmental Biology, University of Michigan, Ann Arbor, \\ Michigan 48105
}

A recently discovered type of mammalian retinal ganglion cell encodes environmental light intensity and mediates non-image-forming visual behaviors, such as the pupillary reflex and circadian photoentrainment. These intrinsically photosensitive retinal ganglion cells (ipRGCs) generate endogenous, melanopsin-based photoresponses as well as extrinsic, rod/cone-driven responses. Because the ipRGCs' light responses and the behaviors they control are both remarkably tonic, these cells have been hypothesized to be capable of irradiance detection lasting throughout the day. I tested this hypothesis by obtaining multielectrode-array recordings from ipRGCs in a novel rat eyecup preparation that enhances the regeneration of rod/cone photopigments. I found that $10 \mathrm{~h}$ constant light could continuously evoke action potentials in these ganglion cells under conditions that stimulated (1) only melanopsin, (2) mainly the rod input, and (3) both intrinsic and extrinsic responses. In response to a $10 \mathrm{~h}$ stimulus with gradual intensity changes to simulate sunrise and sunset, ipRGC firing rates slowly increased during the "sunrise" phase and slowly decreased during the "sunset" phase. Furthermore, I recorded from putative ipRGCs of melanopsin-knock-out mice and found that these cells retained the ability to respond in a sustained fashion to 20 min light steps, indicating that melanopsin is not required for such tonic responses. In conclusion, ipRGCs can signal light continuously for at least $10 \mathrm{~h}$ and can probably track gradual irradiance changes over the course of the day. These results further suggest that the photoreceptors and $\mathrm{ON}$ bipolar cells presynaptic to ipRGCs may be able to respond to light continuously for $10 \mathrm{~h}$.

\section{Introduction}

The retina analyzes four aspects of our visual world: form, color, motion, and irradiance. Whereas the first three types of analysis give rise to image-forming visual perception, long-term irradiance coding underlies non-image-forming visual responses, such as the pupillary reflex, the suppression of nocturnal melatonin release, circadian photoentrainment, and the acute inhibition of the locomotor activity of nocturnal mammals ("negative masking"). Retinal ganglion cells (RGCs) specializing in long-term irradiance detection generate light responses whose spike frequencies stably encode the overall ambient light level. Such RGCs were first recorded by Barlow and Levick (1969), who called them "luminance units." Three decades later, Pu $(1999,2000)$ showed that at least some of these luminance units could be retrogradely labeled from the hypothalamic suprachiamastic nucleus (SCN), site of the master circadian clock. Shortly afterward, Saper, Berson, Yau, and colleagues discovered that these SCN-projecting RGCs express the novel photopigment melanopsin (Provencio et al., 1998) and function as photoreceptors, and thus named them "intrinsically

\footnotetext{
Received March 22, 2012; revised June 22, 2012; accepted July 3, 2012.

Author contributions: K.Y.W. designed research; K.Y.W. performed research; K.Y.W. analyzed data; K.Y.W. wrote the paper.

This work was funded by a Scientific Career Development Award from Research to Prevent Blindness, National Institutes of Health Grant R00 EY18863, and the Kellogg Eye Center Core Grant P30 EY007003. I thank Samer Hattar for the melanopsin-knock-out mice, Austra Liepa for animal husbandry, and Christine Crilly for secretarial support. The author declares no competing financial interests.

Correspondence should be addressed to Kwoon Y. Wong, at the above address. E-mail: kwoon@umich.edu. DOI:10.1523/JNEUROSCI.1423-12.2012

Copyright $\odot 2012$ the authors $\quad 0270-6474 / 12 / 3211478-08 \$ 15.00 / 0$
}

photosensitive retinal ganglion cells" or "ipRGCs" (Gooley et al., 2001; Berson et al., 2002; Hattar et al., 2002). Subsequent work found that even though the ganglion-cell photoreceptors can respond directly to light, they nonetheless receive synaptic input (mainly from the ON channel) and every ipRGC generates rod/cone-mediated as well as melanopsin-based light responses. Both response components are remarkably sustained and are far more tonic than the responses of conventional ganglion cells (Dacey et al., 2005; Wong et al., 2007), suggesting that ipRGCs engage in prolonged irradiance measurement.

Considering the sustained nature of ipRGC photoresponses and of non-image-forming visual behaviors, these ganglion cells have been thought to be capable of continuously signaling light over very long time scales (e.g., throughout the day). However, this hypothesis remained untested because the longest light stimuli that had been attempted on ipRGCs of adult animals were only 20 min of static light (Berson et al., 2002) and 22 min of progressively dimming or brightening light (Barlow and Levick, 1969). Because ganglion-cell photoreceptors exhibit pronounced adaptation in the face of continued photic stimulation (Wong et al., 2005), it is conceivable that their light responses would terminate after only several hours of light exposure. Indeed, a recent study found that the light-induced firing rates of neonatal ipRGCs decayed by $>90 \%$ after just $1 \mathrm{~h}$ of photic stimulation (Sexton et al., 2012), although adult ipRGCs were not examined in that study. Another unresolved question was whether melanopsin is required for very prolonged irradiance detection. Two studies seemed to suggest it is. In the first study, when a $3 \mathrm{~h}$ light was presented to mice during their subjective night, wild-type animals exhibited negative masking for the entire stimulus duration, but the 


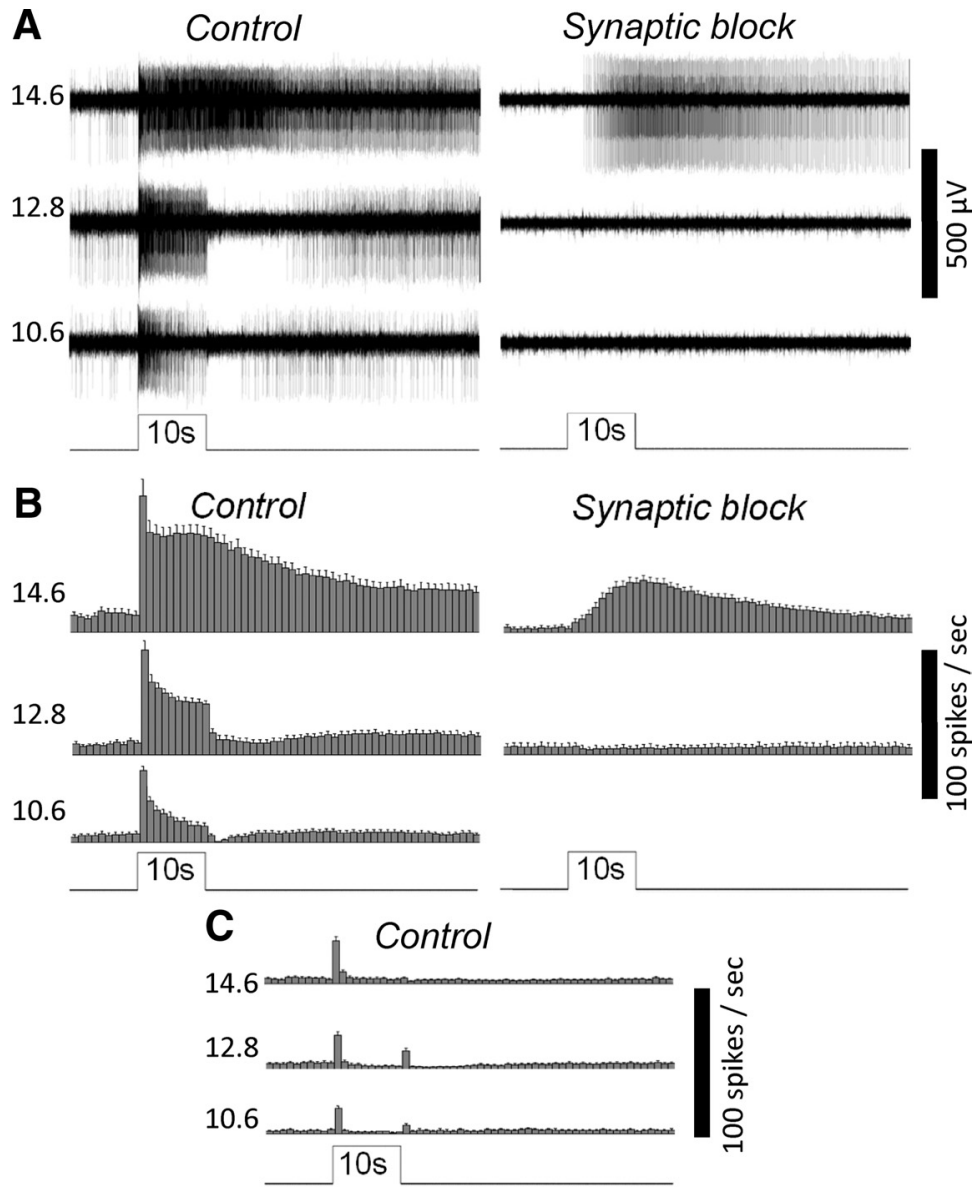

Figure 1. MEA recordings of the novel rat eyecup preparation can detect the light responses of ipRGCs. $A$, The responses of an ipRGC to $10 \mathrm{~s} 480 \mathrm{~nm}$ light steps of three different intensities (expressed in log quanta $\mathrm{cm}^{-2} \mathrm{~s}^{-1}$ ), first in normal Ames' medium (Control) and then in the presence of $\mathrm{L}-(+)-2$-amino-4-phosphonobutyric acid, D-(-)-2-amino-5-phosphonopentanoic acid, and 6,7-dinitroquinoxaline-2,3-dione to block rod/cone signaling (Synaptic block). This ganglion cell was identified as an ipRGC based on its ability to generate sluggish, high-threshold photoresponses during synaptic block, indicative of melanopsin phototransduction. $\boldsymbol{B}$, Spike histograms illustrating the averaged light responses of all the ipRGCs detected in this experiment $(n=18)$. The bin size of these histograms is $1 \mathrm{~s}$, and the error bars represent SEM. C, The averaged light responses of all the conventional ON and ON-OFF ganglion cells analyzed in this experiment $(n=29)$.

masking responses of melanopsin-knock-out mice decayed rapidly and disappeared completely within $2 \mathrm{~h}$ (Mrosovsky and Hattar, 2003). In the second study, SCN neurons in wild-type mice were found to generate sustained light-evoked responses, whereas those in melanopsin-knock-out mice responded to light more transiently (Mure et al., 2007). The present investigation was conducted to examine both questions.

\section{Materials and Methods}

Animals

All experimental procedures were approved by the University Committee on Use and Care of Animals at the University of Michigan. All animals were housed in a $12 \mathrm{~h}$ light, $12 \mathrm{~h}$ dark environment where the lights were on from 6:00 A.M. to 6:00 P.M. The rats used in this study were Long-Evans rats between $\sim 2$ and 7 months of age. The mice were the melanopsin::Cre mice described by Ecker et al. (2010), which had mixed BL/6 and 129Sv/J backgrounds and were $\sim 6$ months of age. For both species, both male and female animals were used.

\section{Multielectrode-array recording}

Rat eyecups. Before each experiment, a rat was kept in a ventilated lightproof box for 15-20 h to fully dark-adapt its ganglion-cell photoreceptors (Wong et al., 2005). Euthanasia and tissue preparation were performed under dim red light. Following euthanasia with carbon diox- ide, both eyes were harvested and hemisected. After most vitreous was removed with forceps, the eyecups were submerged in roomtemperature Ames' medium bubbled with air containing $95 \% \mathrm{O}_{2}$ and $5 \% \mathrm{CO}_{2}$. A square piece of the eyecup measuring $\sim 2-2.5 \mathrm{~mm}$ on each side was cut out with a razor blade and flattened with the ganglion cell side down onto a 60-channel multielectrode array (MEA, 30 $\mu \mathrm{m}$-diameter titanium nitride electrodes at a $200 \mu \mathrm{m}$ center-to-center spacing; Multi Channel Systems). To anchor the preparation, a flat nylon mesh net (Warner Instruments) was placed on top of the scleral side and this net was in turn held down by a platinum ring. The bathing solution was Ames' medium supplemented with $16 \mathrm{~mm}$ D-glucose and maintained at $33^{\circ} \mathrm{C}$ with a temperature controller (Multi Channel Systems). This solution was fed into the recording chamber at $3-7 \mathrm{ml} \mathrm{min}^{-1}$ and was recycled using a peristaltic pump. To ensure adequate oxygenation of the retina, air containing $95 \% \mathrm{O}_{2}$ and $5 \% \mathrm{CO}_{2}$ was continuously applied to Ames' medium in the MEA chamber as well as in the solution reservoir. For the experiments described in Figures 1 and 2, right, the eyecup preparations were superfused in the dark for $2-4 \mathrm{~h}$ before data acquisition started. For the experiments shown in Figures 3 and 4 , the preparations were superfused in darkness for $12-20 \mathrm{~h}$ before data acquisition; such a long preexperiment superfusion gave spike amplitudes time to settle to a reasonably steady state, which was important for the $11.5 \mathrm{~h}$ recordings shown in Figures 3 and 4.

Isolated mouse retinas. The procedures for dark adaptation, euthanasia, enucleation, and hemisection were identical to those described above for rats. After hemisection, the retinas were isolated from the RPE, and forceps were used to remove most vitreous from the retinas. One of the retinas was cut in half, and one piece was flattened on an MEA with the ganglion cell side down. The methods for anchoring the preparation, superfusion, and temperature regulation were the same as those for rats (see above). Each mouse preparation was superfused for $\sim 1$ $\mathrm{h}$ before data acquisition began.

Electrophysiological recording and light stimulation. Extracellular spike recordings were obtained from ganglion cells using a 60-channel MEA amplifier (Multi Channel Systems) (Tu et al., 2005). The recordings had a bandwidth of $200 \mathrm{~Hz}-3 \mathrm{kHz}$ and sampling frequencies were $5-10 \mathrm{kHz}$. Raw recordings from all 60 electrodes were saved onto a computer for offline processing. Light stimuli were full-field $480 \mathrm{~nm}$ light generated by a monochromator with $16 \mathrm{~nm}$ slit width (Optical Building Blocks), and the timing of stimulus presentation was controlled by an electromechanical shutter integrated into this monochromator. Light intensity was adjusted by a continuously variable neutral density filter (Newport) positioned adjacent to the monochromator output, and was calibrated using a radiometer (UDT Instruments). All light intensities shown in the figures are expressed in log quanta $\mathrm{cm}^{-2} \mathrm{~s}^{-1}$. After the light had transmitted through the neutral density filter, it was delivered via a fiber optic cable to the retinal preparation through the bottom of the MEA chamber. Light stimuli were typically presented between 6:00 A.M. and 8:00 P.M. [i.e., mainly during the animals' subjective day (see Animals)]. For the experiment shown in Figure 1, ipRGCs were identified as the units that remained photosensitive during bath application of a mixture of synaptic blockers (see Chemicals). For all subsequent experiments, a 1 min $15.6 \log$ quanta $\mathrm{cm}^{-2}$ $\mathrm{s}^{-1}$ light step was presented at the end of each experiment to identify 


\section{Isolated retinas}

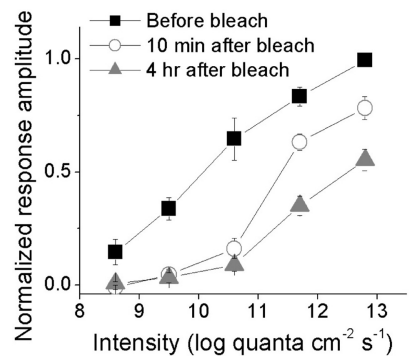

Eyecups

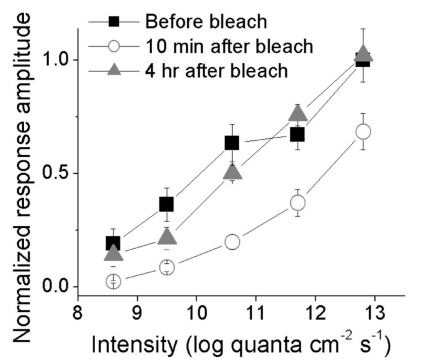

Figure 2. The rat eyecup preparation improves the postbleach recovery of rod/conemediated ipRGC light responses. In this experiment, an intensity series (8.6-12.8 log quanta $\mathrm{cm}^{-2} \mathrm{~s}^{-1}$ ) of $10 \mathrm{~s}$ light steps was presented three times to every ipRGC, first during the dark-adapted state, then $\sim 10 \mathrm{~min}$ after the presentation of a 1 min 13.8 log quanta $\mathrm{cm}^{-2} \mathrm{~s}^{-1}$ light to bleach rod/cone photopigments, and finally $\sim 4 \mathrm{~h}$ after this bleaching light. These plots are averages of all the ipRGCs tested, with each cell's highest-amplitude light response normalized to 1 . For the ipRGCs in isolated retinas ( $n=9$; left), their responses recorded $10 \mathrm{~min}$ after the bleach were somewhat larger those obtained $\sim 4 \mathrm{~h}$ later, indicating not only a lack of $\mathrm{rod} / \mathrm{cone}$ photopigment regeneration but also a gradual rundown between these time points. By contrast, the light responses of ipRGCs in eyecups $(n=9$; right) increased during these two time points, suggesting the RPE enabled some degree of photopigment regeneration during dark adaptation.

ipRGCs as the units continuously excited by this light step; all other ganglion cells responded transiently (Figs. 1C).

Action potentials were sorted offline using the Plexon Offline Sorter (Plexon) and analyzed using Microcal Origin software (OriginLab). In the experiment illustrated in Figure 2, photoresponse amplitude was calculated by subtracting the mean firing rate during the $10 \mathrm{~s}$ prestimulus period from that during the $10 \mathrm{~s}$ light stimulus, and each ipRGC's largest response was normalized to 1. For the histograms shown in Figures 3 and 4 , only the ganglion-cell photoreceptors whose spike waveforms remained sufficiently stable throughout the $11.5 \mathrm{~h}$ recordings were included. Over the course of the $11.5 \mathrm{~h}$, most ipRGCs' action potential waveforms fluctuated to such an extent that sorting became extremely difficult if not impossible. On the other hand, some degree of spike amplitude fluctuation is expected, since ipRGC action potentials tend to get smaller as spike frequency increases (Figs. 4, 5A; Wong et al., 2005). To generate the plot shown in Fig. $3 C$, each cell's baseline firing rate was assumed to remain constant throughout the recording. The total spike count during the $0.5 \mathrm{~h}$ prestimulation period was first multiplied by 23 to estimate the total number of spontaneous spikes during the $11.5 \mathrm{~h}$ recording. This value was then subtracted from the total spike count for the entire recording to give the number of light-induced spikes. In all figures, error bars depict SEM.

\section{Whole-cell recording}

Isolated mouse retinas. The procedures for dark adaptation, euthanasia, enucleation, hemisection, retinal isolation, and vitrectomy were the same as those described above for MEA recording. One of the retinas was cut in half and one piece was flattened on the glass bottom of a superfusion chamber (Warner Instruments) with the ganglion cell side up. This piece was held down by a weighted net (Warner Instruments). The bathing solution was Ames' medium supplemented with $16 \mathrm{~mm}$ D-glucose and maintained at $32^{\circ} \mathrm{C}$ with a temperature controller (Warner Instruments). The bathing solution was fed into the recording chamber at $2-3 \mathrm{ml}$ $\min ^{-1}$ and was recycled using a peristaltic pump.

Electrophysiological recording and light stimulation. Whole-cell recordings were made from randomly selected ganglion cells in isolated, flatmounted retinas harvested from melanopsin-knock-out mice (Ecker et al., 2010). The chamber was mounted on a fixed-stage upright microscope (Nikon Eclipse FN1). The ganglion-cell layer was visualized by infrared transillumination, and medium and large somas were targeted for whole-cell recording. Glass micropipettes were pulled from thickwalled borosilicate tubing (tip resistances 6-8 M 2 ) on a Narishige PC-10 puller and were filled with an intracellular solution that contained the fluorescent dye Lucifer yellow (see Chemicals). Recordings were made in current-clamp mode with a Multiclamp 700B amplifier (Molecular Devices). pCLAMP 10 (Molecular Devices) was used for data acquisition. Signals were low-pass filtered at $2.4 \mathrm{kHz}$ and sampled at $10 \mathrm{kHz}$. Light stimuli were full-field $480 \mathrm{~nm}$ light, generated by filtering the microscope's tungsten-halogen lamp with a narrowband filter $(\sim 20 \mathrm{~nm}$ at half height). All stimuli were introduced from below the superfusion chamber's glass bottom and had an intensity of $12.5 \log$ quanta $\mathrm{cm}^{-2} \mathrm{~s}^{-1}$ at the retina. A logic-controlled electromechanical shutter regulated stimulus timing. Immediately after recording, Lucifer yellow staining of the cell was visualized and depth of dendritic stratification within the inner plexiform layer was estimated by through-focus microscopy. Drawings of stained cells were made using a camera lucida.

\section{Chemicals}

In the whole-cell recording experiments, the intracellular solution contained the following (in mM): $120 \mathrm{~K}$-gluconate; $5 \mathrm{NaCl} ; 4 \mathrm{KCl} ; 10 \mathrm{HEPES}$; 2 EGTA; 4 ATP-Mg; 0.3 GTP-Tris; 7 phosphocreatine-Tris; 0.1\% Lucifer yellow. The $\mathrm{pH}$ of this solution was adjusted to 7.3 with $\mathrm{KOH}$. For the MEA experiments in which rod/cone signaling to the inner retina was blocked, the Ames' medium contained a mixture of glutamate analogs to block synaptic transmission: L-(+)-2-amino-4-phosphonobutyric acid $(100 \mu \mathrm{M}), 6,7$-dinitroquinoxaline-2,3-dione $(80-160 \mu \mathrm{M})$, and D-(-)-2amino-5-phosphonopentanoic acid $(25-50 \mu \mathrm{M})$. These glutamate analogs were purchased from Tocris Bioscience. All other chemicals were purchased from Sigma-Aldrich.

\section{Results}

\section{A novel retinal preparation for studying prolonged} light responses

To properly investigate the responses of ganglion cells to many hours of illumination, a new retinal preparation was needed because all the methods available had significant limitations. MEA recording can remain reasonably stable for hours, but recordings from rodents always used isolated retinas, which do not support the regeneration of rhodopsin following photobleaching. While whole-cell recordings can be made from eyecups in which the retina is attached to the retinal pigment epithelium (RPE) so that rhodopsin regeneration can occur, stable recordings lasting for $>2 \mathrm{~h}$ are extremely rare. To overcome these limitations, I developed a novel recording method in which a small piece of a rat eyecup is flattened on a 60-electrode MEA with the ganglion cell side down. In this preparation, each piece of eyecup typically yielded 3-10 well isolated ipRGCs, which were identified based on their persisting photosensitivity in the presence of synaptic blockers (see Materials and Methods, Chemicals; Wong et al., 2007). The responses of these ganglion cells to $10 \mathrm{~s}$ full-field light steps resembled those recorded previously using isolated rat retinas (Wong et al., 2007). Specifically, every ipRGC generated both on-excitatory, rod/cone-driven ("extrinsic") light responses and melanopsin-mediated ("intrinsic") photoresponses in normal Ames' medium, but only intrinsic responses during synaptic block. These intrinsic photoresponses were evoked only by relatively bright light and had very slow kinetics, with spike frequency increases that started several seconds after stimulus onset and that continued for many seconds after stimulus offset. By contrast, the extrinsic light responses were several log units more sensitive than the melanopsin responses and consisted of spike rate increases that started nearly instantaneously at light onset, continued throughout the $10 \mathrm{~s}$ light, and terminated rapidly after stimulus termination (Fig. 1A,B). The light-on responses of $\mathrm{ON}$ and $\mathrm{ON}-\mathrm{OFF}$ conventional ganglion cells (which were identified by their lack of photosensitivity during synaptic block) also matched those recorded previously using isolated rat retinas (Wong et al., 2007). Namely, they were far more transient than 


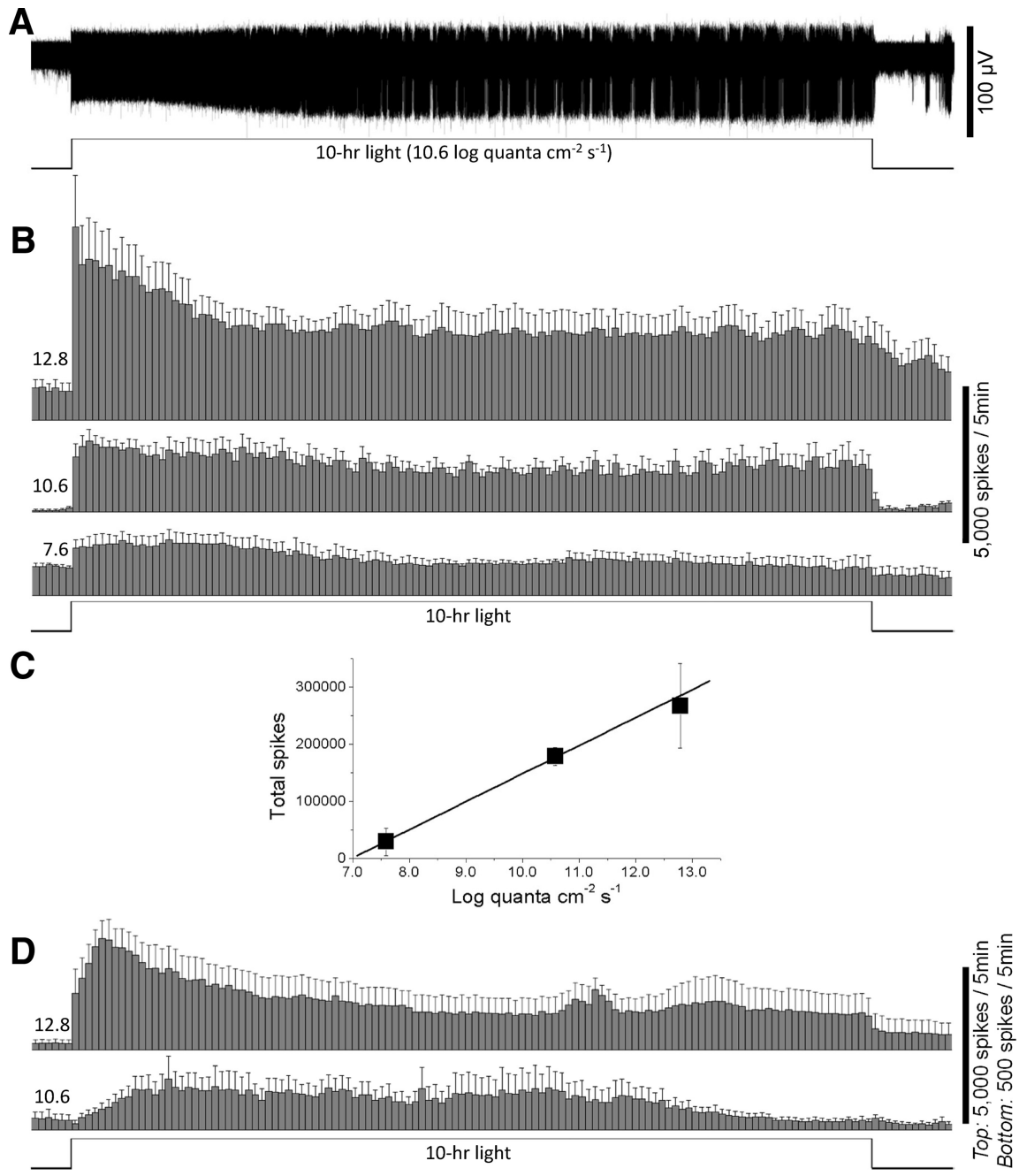

Figure 3. The ipRGCs can spike continuously in response to $10 \mathrm{~h}$ step increases in light intensity. $A$, The response of an ipRGC to a $10 \mathrm{~h} 10.6 \log$ quanta $\mathrm{cm}^{-2} \mathrm{~s}^{-1}$ light step, recorded in normal Ames' medium to allow rod/cone signaling. $\boldsymbol{B}$, Averaged spike histograms of all the ipRGCs recorded in normal Ames' medium, with a bin size of $5 \mathrm{~min}$. The number of cells that contributed to these histograms was six for $12.8 \log$ quanta $\mathrm{cm}^{-2} \mathrm{~s}^{-1}$, three for $10.6 \log$ quanta $\mathrm{cm}^{-2} \mathrm{~s}^{-1}$, and three for $7.6 \log$ quanta $\mathrm{cm}^{-2}$ $s^{-1}$. C, Total spike counts in the $10 \mathrm{~h}$ responses recorded in normal Ames' medium were estimated (see Materials and Methods) and plotted versus light intensity. In the linear regression fit, $r$ equals 0.999 and the slope is 49,039 spikes per log unit. $\boldsymbol{D}$, Averaged spike histograms of all the ipRGCs tested in the presence of synaptic blockers to isolate melanopsin photoresponses, with a bin size of $5 \mathrm{~min}$. The number of cells used in these histograms was four for $12.8 \log$ quanta $\mathrm{cm}^{-2} \mathrm{~s}^{-1}$ and four for $10.6 \log$ quanta $\mathrm{cm}^{-2}$ $s^{-1}$. Notice that the response amplitude scale bars are different for the two intensities.

the ipRGCs' extrinsic photoresponses, with spike rate elevations that never lasted for $>\sim 3 \mathrm{~s}$ (Fig. 1C). This result showed that any ganglion cell that could respond continuously to constant light for at least $10 \mathrm{~s}$ could be safely assumed to be an ipRGC. In all the experiments described below, ganglion-cell photoreceptors were identified as the cells that could continuously generate spiking responses to a $1 \mathrm{~min} 15.6 \log$ quanta $\mathrm{cm}^{-2} \mathrm{~s}^{-1}$ light step presented at the end of each experiment.

The experiment shown in Figure 2 confirmed that this new preparation enhances the extent of postbleach photopigment regeneration. In this experiment, the responses of ipRGCs to an intensity series of $10 \mathrm{~s}$ light were first recorded in the darkadapted state. An intense $480 \mathrm{~nm}$ light $\left(13.8 \log\right.$ quanta $\mathrm{cm}^{-2}$ $\mathrm{s}^{-1}$ ) was then presented for $1 \mathrm{~min}$ to bleach most rod/cone photopigment molecules. Following the bleach, the same intensity series of $10 \mathrm{~s}$ light was presented after 10-18 min of dark adaptation, and then again after $\sim 4 \mathrm{~h}$ of additional dark adaptation. In both preparations, all light responses recorded 10-18 min after the bleach were significantly reduced compared with the prebleach recordings. For the isolated retinas, response amplitudes declined further $4 \mathrm{~h}$ after the bleach, indicating not only a lack of photopigment regeneration but also a gradual rundown in photosensitivity. In contrast, the light responses of all ipRGCs in eyecups increased in amplitude between the two time points of dark adaptation, indicating substantial regeneration of $\mathrm{rod} /$ cone pigments.

\section{The ipRGCs can continuously respond to 10 hours of constant illumination} The next set of experiments examined the responses of ganglion-cell photoreceptors to prolonged photic stimulation. Following 12-20 h of dark adaptation (see Materials and Methods), each rat eyecup superfused with normal Ames' medium was presented with a $10 \mathrm{~h}$ full-field step increase in light intensity. Four intensities were tested: $12.8 \log$ quanta $\mathrm{cm}^{-2} \mathrm{~s}^{-1}, \sim 1$ log unit above the melanopsin threshold for the responses of mouse and primate ipRGCs to 1 min light steps (Dacey et al., 2005; Tu et al., 2005); 10.6 log quanta $\mathrm{cm}^{-2} \mathrm{~s}^{-1}$, which approximately corresponds to the threshold for cone input to primate ipRGCs (Dacey et al., 2005); 7.6 $\log$ quanta $\mathrm{cm}^{-2} \mathrm{~s}^{-1}$, just above the threshold for the rod input to ipRGCs in RPE-attached primate retinas (Dacey et al., 2005); and $6.7 \log$ quanta $\mathrm{cm}^{-2} \mathrm{~s}^{-1}$, which is just below the aforementioned rod input threshold. The $6.7 \log$ stimulus failed to elicit any response $(n=13)$, whereas all the other three intensities evoked ipRGC firing throughout the $10 \mathrm{~h}$ light step after a short onset latency (Fig. $3 A, B)$. At all three suprathreshold intensities, an appreciable increase in firing was observed within $2 \mathrm{~s}$ of light stimulation. After the responses had peaked, firing rates gradually decayed toward a plateau that remained fairly stable over the remainder of the $10 \mathrm{~h}$ stimulus (Fig. $3 B$ ). On the other hand, at light offset, the rates at which spike frequencies returned to baseline levels depended on light intensity. At 7.6 log quanta $\mathrm{cm}^{-2} \mathrm{~s}^{-1}$, firing rates fully returned to baseline levels within $\sim 10 \mathrm{~s}$ for all cells. At $10.6 \log$ quanta $\mathrm{cm}^{-2} \mathrm{~s}^{-1}$, spike frequencies decayed to baseline over several minutes, whereas at $12.8 \log$ quanta $\mathrm{cm}^{-2} \mathrm{~s}^{-1}$, firing remained elevated for at least $1 \mathrm{~h}$ (Fig. 3B). In Figure 3C, the spike counts of all the responses to the three suprathreshold intensities were estimated (see Materials and Methods) and plotted against photon flux, showing that the number of light-evoked spikes increased more or less linearly as intensity increased.

To selectively investigate the intrinsic melanopsin response to $10 \mathrm{~h}$ light steps, the next experiment was conducted in the presence of synaptic blockers (see Materials and Methods). Three intensities were examined: $12.8,10.6$, and $9.5 \log$ quanta $\mathrm{cm}^{-2}$ $\mathrm{s}^{-1}$. As mentioned above, the $12.8 \log$ stimulus is $\sim 1 \log$ unit 
above the melanopsin threshold previously measured using 1 min light steps (Dacey et al., 2005; Tu et al., 2005), whereas the 10.6 and $9.5 \log$ stimuli are both below that threshold. I found that $12.8 \log$ quanta $\mathrm{cm}^{-2} \mathrm{~s}^{-1}$ robustly evoked action potentials throughout the $10 \mathrm{~h}$ stimulation. This intensity started to induce an appreciable increase in spiking within $\sim 20$ s of stimulation. The responses reached peak amplitudes within approximately half an hour, and then gradually decayed toward a steady-state plateau. After stimulus termination, spike rates dropped gradually over many minutes (Fig. 3D, top). I also found that 10.6 $\log$ quanta $\mathrm{cm}^{-2} \mathrm{~s}^{-1}$ evoked melanopsin responses for $10 \mathrm{~h}$. At this intensity, appreciable firing increases were observed only after 2-20 min of light stimulation. Spike frequencies rose for many minutes and peak amplitudes were reached $\sim 1 \mathrm{~h}$ after stimulus onset. In the averaged response shown in Figure $3 D$, bottom, the response may appear to have fully decayed to baseline after $\sim 8-9$ h of light stimulation; however, shortly after light off, spike frequency obviously drops further, suggesting that the cells were still responding to the stimulus at the end of the $10 \mathrm{~h}$ light step. On the other hand, the $9.5 \log$ quanta $\mathrm{cm}^{-2} \mathrm{~s}^{-1}$ stimulus did not evoke any obvious melanopsin response in any of the ipRGCs tested $(n=19)$.

\section{ipRGC spiking can approximately track continuously changing light intensities}

All the light stimuli tested so far were step increases in light intensity. However, under natural conditions, most animals and many people experience gradual rises in ambient light intensity during the morning and gradual reductions in the evening (Hébert et al., 1998). To examine how ganglion-cell photoreceptors might respond to gradual irradiance changes, I recorded from rat eyecups superfused with normal Ames' medium and presented a $10 \mathrm{~h}$ light stimulus that consisted of a $2.5 \mathrm{~h}$ ramp increase in light intensity from $8.1-12.8 \mathrm{log}$ quanta $\mathrm{cm}^{-2} \mathrm{~s}^{-1}$ to simulate sunrise, followed by $5 \mathrm{~h}$ of constant intensity at $12.8 \log$ quanta $\mathrm{cm}^{-2} \mathrm{~s}^{-1}$, and subsequently a $2.5 \mathrm{~h}$ ramp decrease to $8.1 \log$ quanta $\mathrm{cm}^{-2} \mathrm{~s}^{-1}$ to mimic sunset [for comparison, in terms of effectiveness in stimulating melanopsin, the irradiance of a typical cloudy day is $\sim 15.6$ $\log$ quanta $\mathrm{cm}^{-2} \mathrm{~s}^{-1}$ of equivalent $480 \mathrm{~nm}$ photons, whereas direct sunlight is $\sim 16.4 \log$ quanta $\mathrm{cm}^{-2} \mathrm{~s}^{-1}$ (Enezi et al., 2011)]. The spike rates of these ganglion cells rose steadily during the "sunrise" phase and peaked at the transition to the constant-intensity phase. Spike frequencies decayed slightly over the $5 \mathrm{~h}$ constant phase, but started to drop faster shortly after the initiation of the "sunset" simulation (Fig. 4).

\section{Melanopsin is not required for sustained ipRGC responses to bright light}

As shown in Figure $3 B$, bottom, ganglion-cell photoreceptors could respond continuously for $10 \mathrm{~h}$ to the $7.6 \log$ quanta $\mathrm{cm}^{-2}$ $\mathrm{s}^{-1}$ constant light. Because this intensity is $\sim 3 \log$ units below the melanopsin threshold measured during synaptic blockade (Fig. $3 D$ ), this result suggested that synaptic input may be sufficient to induce sustained ipRGC responses to very dim light. To examine whether synaptic input is sufficient for prolonged ipRGC responses to bright light, I obtained whole-cell and MEA record- ings from melanopsin-knock-out mice, specifically the melanopsin::Cre mice created by Ecker et al. (2010) in which both melanopsin promoters drive the expression of the Cre recombinase instead of the melanopsin open reading frame.

In the whole-cell recording experiment, current-clamp recordings were made from 15 randomly selected ganglion cells in melanopsin-knock-out retinas. I presented $480 \mathrm{~nm}$ light steps $10-60 \mathrm{~s}$ in duration and $12.5 \log$ quanta $\mathrm{cm}^{-2} \mathrm{~s}^{-1}$ in intensity to each cell and only 2 of the 15 cells responded in a sustained manner (Fig. 5A). Lucifer yellow fills revealed that both cells' morphologies were strikingly similar to those of ipRGCs. The first cell, shown in Figure $5 A$, left, had two strata of relatively sparse dendrites and was thus probably an M3-type ipRGC (Schmidt and Kofuji, 2011). The second cell, shown in Figure 5A, right, had a very large soma and relatively dense, radiate dendrites stratifying near the retinal surface, indicating it was likely an M4type ipRGC (Ecker et al., 2010). These results suggest that, even without melanopsin, presumed ipRGCs can still respond continuously to relatively bright light for at least $1 \mathrm{~min}$. They further suggest that in melanopsin-knock-out mouse retinas, sustained photoresponses are probably a unique characteristic of ipRGCs, just like for wild-type rat retinas (Fig. 1).

To investigate the responses of ipRGCs to longer stimulus durations and to obtain a larger sample size, I made MEA recordings from melanopsin-knock-out mouse retinas. This experiment used isolated retinas, partly because I have found it impossible to flatten mouse eyecups onto MEAs and partly because vitrectomy (which is necessary to allow the MEA to contact the retinal surface) invariably causes mouse retinas to detach from the RPE. For all four pieces of retinas tested, the vast majority of MEA channels exhibited only transient light responses (Fig. $5 B$ ), and each 60-channel MEA contained only several well isolated units with sustained photoresponses (Fig. 5C, top and middle). This overwhelming prevalence of transient units paralleled that observed in the above-mentioned whole-cell recordings and in MEA recordings of wild-type retinas, suggesting that the transient cells were likely conventional RGCs, whereas the sustained responders were probably ipRGCs. In conclusion, in these melanopsin-knock-out retinas, putative ipRGCs could still be identified by their continuous spiking responses to the $1 \mathrm{~min} 15.6$ $\log$ quanta $\mathrm{cm}^{-2} \mathrm{~s}^{-1}$ test pulse (see Materials and Methods), as shown in Figure 5, top and middle. When rod/cone input was blocked using the synaptic blockers, all responses to this test pulse were abolished, confirming that these presumed ipRGCs lacked melanopsin (Fig. 5C, bottom). To test these cells' ability to signal 
A

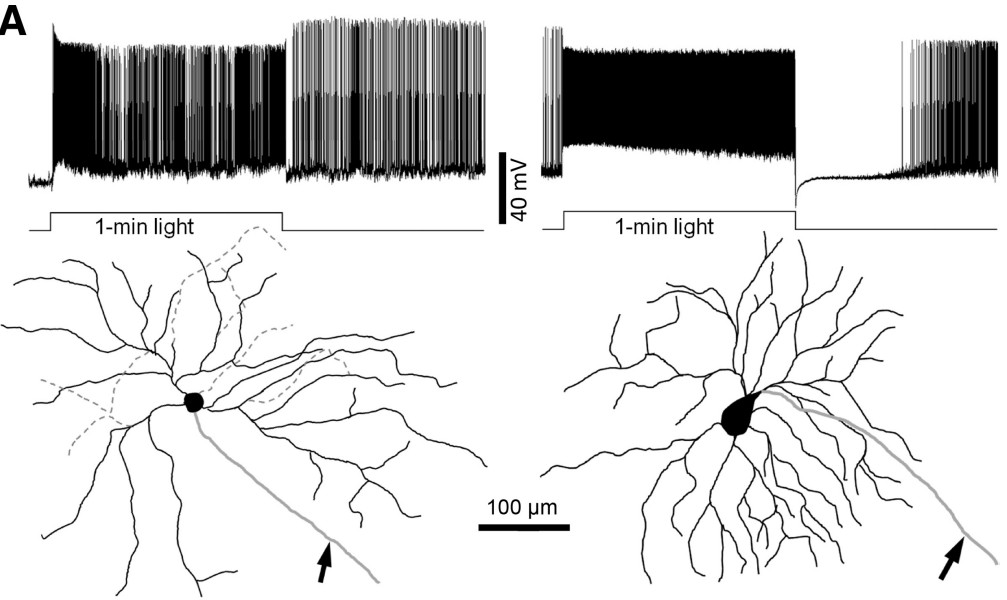

B
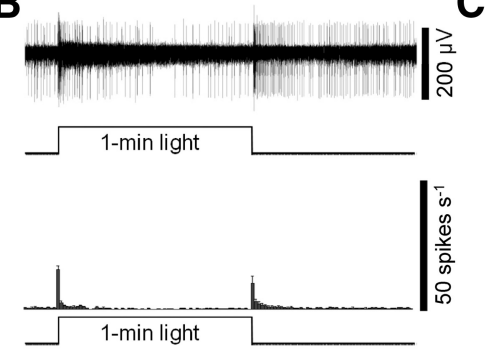

C

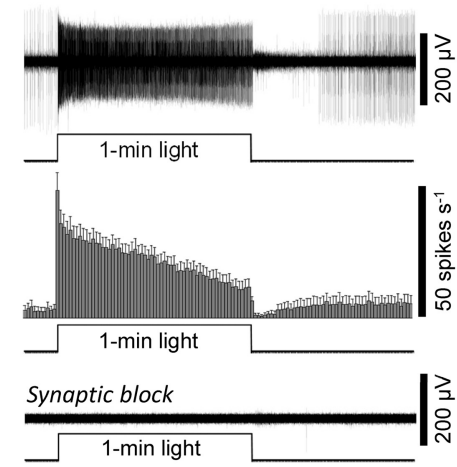

D

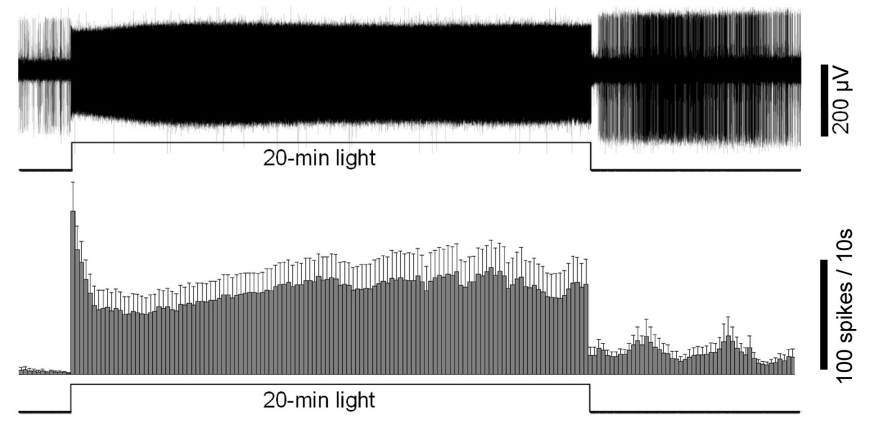

Figure 5. Melanopsin is not required for prolonged ipRGC responses to bright light. $\boldsymbol{A}$, Whole-cell recordings showed that in melanopsin-knock-out retinas, ganglion cells with ipRGC-like morphologies could respond continuously to bright light for $1 \mathrm{~min}$. Left, The response of a sustained ON ganglion cell to a $1 \mathrm{~min} 12.5 \mathrm{log}$ quanta $\mathrm{cm}^{-2} \mathrm{~s}^{-1}$ light step (top) and its M3-like morphology (bottom). The solid black lines depict dendrites near the retinal surface, whereas the dashed gray lines depict deeper dendrites. Right, The response of another sustained $\mathrm{ON}$ ganglion cell to the same 1 min stimulus (top) and its M4-like morphology (bottom). Both cells' axons are indicated by arrows. B, C, For each piece of melanopsin-knock-out mouse retina recorded on the MEA, the vast majority of channels exhibited only transient photoresponses and only several channels contained sustained responses. Thus, the transient units were probably conventional RGCs while the sustained ones were probably ipRGCs. $\boldsymbol{B}$, Top, The transient response of a presumed conventional RGC to a $1 \mathrm{~min} 15.6 \log$ quanta $\mathrm{cm}^{-2} \mathrm{~s}^{-1}$ light step, recorded in normal Ames' medium. Bottom, A histogram averaging the spiking responses of 30 transient $0 \mathrm{~N}$ or $0 \mathrm{~N}-0 \mathrm{FF} R G C \mathrm{C}$ to this $1 \mathrm{~min}$ light step, with a bin size of $1 \mathrm{~s}$. C, Top, The response of a putative melanopsin-knock-out ipRGC to a $1 \mathrm{~min} 15.6 \log$ quanta $\mathrm{cm}^{-2} \mathrm{~s}^{-1}$ light step in normal Ames' medium. Middle, A histogram averaging the spiking responses of all the presumed melanopsin-knock-out ipRGCs to this 1 min light step, with a bin size of $1 \mathrm{~s}(n=13)$. Bottom, During superfusion with synaptic blockers, the light response of the cell shown in $C$, top, was completely abolished, confirming the lack of intrinsic photosensitivity. D, Synaptic input to putative melanopsin-knock-out ipRGCS could evoke action potentials throughout a $20 \mathrm{~min} 12.8 \mathrm{log}$ quanta $\mathrm{cm}^{-2} \mathrm{~s}^{-1}$ light step. Top, The response of one of these cells, recorded in normal Ames' medium. Bottom, A spike histogram averaging all the putative melanopsin-knock-out ipRGCs' responses to the $20 \mathrm{~min} 12.8 \log$ quanta $\mathrm{cm}^{-2} \mathrm{~s}^{-1}$ light, with a bin size of $10 \mathrm{~s}(n=13)$.

prolonged bright light, a $12.8 \log$ quanta $\mathrm{cm}^{-2} \mathrm{~s}^{-1}$ light was presented for $20 \mathrm{~min}$. I used such a short duration because MEA recordings of isolated mouse retinas have far shorter longevity than those of rat eyecups and because rhodopsin regeneration does not occur in the absence of the RPE. Nearly all putative
ipRGCs (12 of 13) were continuously excited throughout this $20 \mathrm{~min} 12.8 \mathrm{log}$ quanta $\mathrm{cm}^{-2} \mathrm{~s}^{-1}$ light. The averaged response peaked rapidly, displayed pronounced adaptation over the first minute, and then slowly increased for the next $\sim 10$ min before reaching a steady level that continued through the end of the 20 min light step. Spike frequencies quickly dropped at light offset, but remained elevated for the remaining $8 \mathrm{~min}$ of the recording (Fig. 5D).

\section{Discussion}

I have developed a novel eyecup preparation that facilitates prolonged recording of rat ganglion-cell light responses. This preparation supports a high degree of recovery from bleaching light (Fig. 2), and robust, stable ganglion-cell photoresponses can be detected for many hours (Figs. 3, 4). In addition to rats, this preparation can probably be applied to other rodents with eyes about the same size or larger. However, the preparation is not suitable for mice due to the difficulty of flattening their small eyes and of removing their vitreous without inducing retinal detachment. Thus, I used rat eyecups for all the experiments described in this paper except when melanopsin was knocked out, which required the use of mice (Fig. 5).

The main finding of this report is that ipRGCs can continuously spike in response to $10 \mathrm{~h}$ of constant light stimulation. Before this study, two lines of evidence had suggested that these ganglion cells might possess such ability. First, behavioral assays showed that negative masking and nocturnal melatonin suppression could be continuously evoked by constant light for 3-6.5 h (Mrosovsky and Hattar, 2003; Gooley et al., 2010). Second, when rats that had been exposed to $19 \mathrm{~h}$ of constant light were killed, the neuronal activity marker cFos was found in ganglion cells that contained pituitary adenylate cyclase activating polypeptide (i.e., putative ipRGCs) but not in ganglion cells that lacked this protein (i.e., presumed conventional RGCs) (Hannibal et al., 2001). However, whereas the light sources used in these studies were constant, the individual photoreceptors of the animals and human subjects likely experienced frequent fluctuations in light intensity. This is because the animals were allowed to move freely in a visually heterogeneous environment and, even though the human subjects were stimulated within Ganzfeld domes, they were allowed to blink their eyes or even take periodic $10 \mathrm{~min}$ breaks from the experiment. An additional caveat of the cFos experiment is that cFos expression does not necessarily indicate spiking activity, which is required for ganglion cells to signal to 
higher brain centers. Thus, the present study provides the first definitive proof that ganglion-cell photoreceptors can spike in response to $10 \mathrm{~h}$ of static photic stimulation, consistent with their roles in long-lasting, non-image-forming visual behaviors. Dacey et al. (2005) demonstrated that for melanopsin responses evoked by 1 min light steps, the number of light-induced spikes is directly proportional to photon flux, indicating that the ipRGC phototransduction cascade counts photons precisely. In the present paper, I found that such a linear relationship also exists for ipRGC responses to $10 \mathrm{~h}$ light steps (Fig. $3 \mathrm{C}$ ), suggesting that these ganglion cells may continue to count photons over many hours. This remarkable photon-counting ability may form the basis for the prolonged integration times characteristic of nonimage-forming visual behaviors (Nelson and Takahashi, 1991).

Another important conclusion from this investigation is that melanopsin is probably not necessary for generating sustained ipRGC photoresponses. Three previous studies showed that $10-15$ s light steps below the melanopsin threshold could continuously excite ipRGCs (Dacey et al., 2005; Wong et al., 2007; Schmidt and Kofuji, 2010). In an electrophysiological study of the photoresponses of SCN neurons in anesthetized mice, SCN cells in wild-type animals were found to respond continuously to 5 min light steps, whereas those in melanopsin-knock-out animals generated relatively transient responses lasting at most 1 min (Mure et al., 2007). Together, these studies appeared to suggest that even though the ipRGCs' extrinsic light responses are more sustained than those of conventional ganglion cells, they can continue for only up to $\sim 1 \mathrm{~min}$ and melanopsin is required for longer photoresponses. A masking study by Mrosovsky and Hattar (2003) reinforced this conclusion by showing that, whereas a $3 \mathrm{~h}$ constant light could continuously suppress the locomotor activity of wild-type mice, its suppressive effect on melanopsin-knock-out animals started to decay within only 20 min. By contrast, in the present study, I obtained two lines of evidence that melanopsin may not be required for tonic ipRGC photoresponses, indicating that the above-mentioned transient SCN and behavioral photoresponses were probably due to synaptic processing occurring downstream from the ipRGCs. First, putative mouse ipRGCs that lacked melanopsin were continuously excited by $20 \mathrm{~min}$ of full-field bright light. Near the end of the 20 min stimulus, these ganglion cells' firing rates showed no sign of decay, suggesting that they could probably have responded for much longer (Fig. 5D). Second, wild-type rat ipRGCs could respond continuously to a $10 \mathrm{~h}$ full-field light step whose intensity was just above the threshold for the rod input and $\sim 3 \log$ units below the melanopsin threshold measured during synaptic block (Fig. $3 B, D$ ). This latter result suggests that rod input to these ganglion cells is probably sufficient to induce at least $10 \mathrm{~h}$ of spiking responses to dim light; however, since melanopsin remained functional in these rat ipRGCs, the possibility that this photopigment contributed to these $10 \mathrm{~h}$ responses could not be ruled out. An implication of these results is that the neurons driving the ipRGCs' extrinsic photoresponses (i.e., the outer retinal photoreceptors and ON bipolar cells) are capable of signaling constant light continuously for at least $20 \mathrm{~min}$ and potentially even $10 \mathrm{~h}$, just like the melanopsin phototransduction cascade. This is somewhat surprising because both the type- 6 metabotropic glutamate receptors on ON bipolar cells and the ionotropic glutamate receptors on ganglion cells exhibit pronounced desensitization (Mittman et al., 1990; Lukasiewicz et al., 1995; Matsui et al., 1998; Nawy, 2004). Desensitization of AMPA/ kainate receptors is particularly significant and their responses to glutamate desensitize nearly completely in $<1 \mathrm{~s}$ (Trussell and
Fischbach, 1989; Lukasiewicz et al., 1995; DeVries and Schwartz, 1999). The present data suggest that during sustained illumination, even though the glutamate receptors presynaptic to the ipRGCs are probably in a desensitized state, desensitization of these receptors does not completely inactivate them, thus allowing very prolonged signaling of light information. By contrast, conventional ganglion cells' spiking responses to full-field light last for only up to several seconds (Fig. $1 C$; Wong et al., 2007). Thus, whereas ipRGCs stably encode irradiance over long time scales, conventional ganglion cells appear to specialize in detecting changes in light intensity. Presumably, the rod/cone photoreceptors' prolonged light responses are truncated by the conventional ganglion cells' presynaptic circuits and/or intrinsic properties.

Using $10 \mathrm{~h}$ light stimulation during synaptic blockade, I determined the threshold for the melanopsin photoresponse to be on the order of $10 \log$ photons $\mathrm{cm}^{-2} \mathrm{~s}^{-1}$ (Fig. 3D, bottom). This is $\sim 1 \log$ unit lower than the melanopsin threshold previously determined using 1 min light steps (Dacey et al., 2005; Tu et al., 2005), and at least 2 log units lower than that measured using $10 \mathrm{~s}$ light (Fig. 1A,B). The likeliest reason for such dependence on stimulus duration is that the melanopsin phototransduction cascade can integrate photons over time scales in excess of $1 \mathrm{~min}$. Thus, while the integration of $10^{10}$ photons $\mathrm{cm}^{-2} \mathrm{~s}^{-1}$ for $1 \mathrm{~min}$ is insufficient to evoke action potentials or even graded depolarizations (Dacey et al., 2005; Tu et al., 2005), much longer integration times can lead to robust spiking responses. Another line of evidence for prolonged photic integration is that at the nearthreshold intensity of $\sim 10.6 \log$ quanta $\mathrm{cm}^{-2} \mathrm{~s}^{-1}$, the intrinsic photoresponses of ipRGCs continued to rise for $>1 \mathrm{~h}$ before reaching the peak amplitude (Fig. 3D, bottom). The melanopsin response developed significantly faster when stimulus intensity was $\sim 2 \log$ units higher, although peak latency was still $\sim 25 \mathrm{~min}$ (Fig. 3D, top). Using responses to single photons, Do et al. (2009) estimated the melanopsin integration time to be only $\sim 40 \mathrm{~s}$, far too brief to account for the peak latencies shown in Figure $3 D$. This short integration time could be partly caused by the fact that Do and colleagues identified ganglion-cell photoreceptors using epifluorescence excitation of fluorescent protein labeling, which severely light-adapted these cells and accelerated their melanopsin response kinetics (Wong et al., 2005).

Previous studies contrasted the fast kinetics of the ipRGCs' extrinsic photoresponses with the sluggish kinetics of these cells' melanopsin responses, showing that the extrinsic responses had nearly instantaneous onset and offset, whereas the intrinsic responses had long onset latencies and prolonged poststimulus persistence (Dacey et al., 2005; Wong et al., 2007). Gamlin and colleagues (2007) further showed that in the primate pupillary reflex, the spectral tuning of the poststimulus sustained response could be well fit by a single vitamin A1-based rhodopsin nomogram with peak sensitivity at $483 \mathrm{~nm}$, close to the $\lambda_{\max }$ for melanopsin $(482 \mathrm{~nm})$. These reports have led some researchers to assume that the poststimulus ipRGC response is solely due to melanopsin. However, in response to bright light, I observed pronounced poststimulus spiking even in mouse ipRGCs lacking melanopsin (Fig. $5 B-D$ ), indicating that, at least under certain conditions, synaptic input can contribute to these ganglion cells' postillumination responses. In agreement with this observation, mouse ON bipolar cells presumed to contact ipRGCs respond to bright light with a depolarization that long outlasts the stimulus (Dumitrescu et al., 2009). 


\section{References}

Barlow HB, Levick WR (1969) Changes in the maintained discharge with adaptation level in the cat retina. J Physiol 202:699-718.

Berson DM, Dunn FA, Takao M (2002) Phototransduction by retinal ganglion cells that set the circadian clock. Science 295:1070-1073.

Dacey DM, Liao HW, Peterson BB, Robinson FR, Smith VC, Pokorny J, Yau KW, Gamlin PD (2005) Melanopsin-expressing ganglion cells in primate retina signal colour and irradiance and project to the LGN. Nature 433:749-754.

DeVries SH, Schwartz EA (1999) Kainate receptors mediate synaptic transmission between cones and 'Off' bipolar cells in a mammalian retina. Nature 397:157-160.

Do MT, Kang SH, Xue T, Zhong H, Liao HW, Bergles DE, Yau KW (2009) Photon capture and signalling by melanopsin retinal ganglion cells. Nature 457:281-287.

Dumitrescu ON, Pucci FG, Wong KY, Berson DM (2009) Ectopic retinal ON bipolar cell synapses in the OFF inner plexiform layer: contacts with dopaminergic amacrine cells and melanopsin ganglion cells. J Comp Neurol 517:226-244.

Ecker JL, Dumitrescu ON, Wong KY, Alam NM, Chen SK, LeGates T, Renna JM, Prusky GT, Berson DM, Hattar S (2010) Melanopsin-expressing retinal ganglion-cell photoreceptors: cellular diversity and role in pattern vision. Neuron 67:49-60.

Enezi J, Revell V, Brown T, Wynne J, Schlangen L, Lucas R (2011) A "melanopic" spectral efficiency function predicts the sensitivity of melanopsin photoreceptors to polychromatic lights. J Biol Rhythms 26:314-323.

Gamlin PD, McDougal DH, Pokorny J, Smith VC, Yau KW, Dacey DM (2007) Human and macaque pupil responses driven by melanopsincontaining retinal ganglion cells. Vision Res 47:946-954.

Gooley JJ, Lu J, Chou TC, Scammell TE, Saper CB (2001) Melanopsin in cells of origin of the retinohypothalamic tract. Nat Neurosci 4:1165.

Gooley JJ, Rajaratnam SM, Brainard GC, Kronauer RE, Czeisler CA, Lockley SW (2010) Spectral responses of the human circadian system depend on the irradiance and duration of exposure to light. Sci Transl Med 2:31 ra33.

Hannibal J, Vrang N, Card JP, Fahrenkrug J (2001) Light-dependent induction of cFos during subjective day and night in PACAP-containing ganglion cells of the retinohypothalamic tract. J Biol Rhythms 16:457-470.

Hattar S, Liao HW, Takao M, Berson DM, Yau KW (2002) Melanopsincontaining retinal ganglion cells: architecture, projections, and intrinsic photosensitivity. Science 295:1065-1070.

Hébert M, Dumont M, Paquet J (1998) Seasonal and diurnal patterns of human illumination under natural conditions. Chronobiol Int 15:59-70.

Lukasiewicz PD, Lawrence JE, Valentino TL (1995) Desensitizing glutamate receptors shape excitatory synaptic inputs to tiger salamander retinal ganglion cells. J Neurosci 15:6189-6199.

Matsui K, Hosoi N, Tachibana M (1998) Excitatory synaptic transmission in the inner retina: paired recordings of bipolar cells and neurons of the ganglion cell layer. J Neurosci 18:4500-4510.

Mittman S, Taylor WR, Copenhagen DR (1990) Concomitant activation of two types of glutamate receptor mediates excitation of salamander retinal ganglion cells. J Physiol 428:175-197.

Mrosovsky N, Hattar S (2003) Impaired masking responses to light in melanopsin-knockout mice. Chronobiol Int 20:989-999.

Mure LS, Rieux C, Hattar S, Cooper HM (2007) Melanopsin-dependent nonvisual responses: evidence for photopigment bistability in vivo. J Biol Rhythms 22:411-424.

Nawy S (2004) Desensitization of the mGluR6 transduction current in tiger salamander On bipolar cells. J Physiol 558:137-146.

Nelson DE, Takahashi JS (1991) Sensitivity and integration in a visual pathway for circadian entrainment in the hamster (Mesocricetus auratus). J Physiol 439:115-145.

Provencio I, Jiang G, De Grip WJ, Hayes WP, Rollag MD (1998) Melanopsin: an opsin in melanophores, brain, and eye. Proc Natl Acad Sci U S A 95:340-345.

Pu M (1999) Dendritic morphology of cat retinal ganglion cells projecting to suprachiasmatic nucleus. J Comp Neurol 414:267-274.

$\mathrm{Pu}$ M (2000) Physiological response properties of cat retinal ganglion cells projecting to suprachiasmatic nucleus. J Biol Rhythms 15:31-36.

Schmidt TM, Kofuji P (2010) Differential cone pathway influence on intrinsically photosensitive retinal ganglion cell subtypes. J Neurosci 30:16262-16271.

Schmidt TM, Kofuji P (2011) Structure and function of bistratified intrinsically photosensitive retinal ganglion cells in the mouse. J Comp Neurol 519:1492-1504.

Sexton TJ, Golczak M, Palczewski K, Van Gelder RN (2012) Melanopsin is highly resistant to light and chemical bleaching in vivo. J Biol Chem 287:20888-20897.

Trussell LO, Fischbach GD (1989) Glutamate receptor desensitization and its role in synaptic transmission. Neuron 3:209-218.

Tu DC, Zhang D, Demas J, Slutsky EB, Provencio I, Holy TE, Van Gelder RN (2005) Physiologic diversity and development of intrinsically photosensitive retinal ganglion cells. Neuron 48:987-999.

Wong KY, Dunn FA, Berson DM (2005) Photoreceptor adaptation in intrinsically photosensitive retinal ganglion cells. Neuron 48:1001-1010.

Wong KY, Dunn FA, Graham DM, Berson DM (2007) Synaptic influences on rat ganglion-cell photoreceptors. J Physiol 582:279-296. 